\title{
Breast tumor detection and classification in Mammograms: Gabor wavelet vs. statistical features
}

\author{
Dharmesh Singh ${ }^{1}$ and Mandeep Singh ${ }^{2 *}$, Vipual Sharma ${ }^{3}$ \\ ${ }^{1}$ Research Scholar, Thapar Institute of Engineering \& Technology, Patiala-147004, India, \\ ${ }^{2}$ Assistant Professor, Thapar Institute of Engineering \& Technology, Patiala-147004, India, \\ ${ }^{3}$ Assistant Professor, IKG Punjab Technical University Bhikhiwind Campus, Punjab, India \\ *corresponding author: \\ Mandeep Singh, Assistant Professor, EIED \\ Thapar Institute of Engineering \& Technology, Patiala-147004, India \\ email : mdsingh@thapar.edu \\ Phone: +91-9815605616
}

\begin{abstract}
Breast cancer is the second cause of fatality among all cancers for women. Automatic classification of breast cancer lesions in mammograms is a challenging task due to the irregularity and complexity of the location, size, shape, and texture of these lesions. The intensity dissimilarity has been found between breast cancer tissues and normal tissues, when a multispectral anatomical mammographic screening scans have been done. In this work, two approaches have been evaluated to classify the breast tumor lesions. The first one is through Gabor wavelet features and the second one is Statistical features. Subsequently, support vector machine, Multilayer Perceptron and KNN classifiers have been used with computer based method for breast tumor classification.
\end{abstract}

Keywords: Lesion classification, statistical features, Gabor wavelet features, mammograms 


\section{Introduction}

Medical imaging is a robust and reliable diagnostic method for the breast related diseases. Breast tumor originates in the internal coating of milk lobules in the breast. It is basically abandoned progress of anomalous cells. A latest summary by National Cancer Registry Programs state that 28-35\% breast cancer from all cancers are spread among females in major cities (Delhi, Mumbai, Chennai etc.) and it is increasing fast in huge figures [1]. The American Cancer Society estimates that around 1,658,370 women in the US will be identified with breast tumor, and due to it about 589,430 females died till 2015 [2]. It is considered that most effective way to raise the chance of save from disease is by diagnosis and treatment in early stages [3-4].

Mammography is the major screening tool which is carried out for detection of breast cancer at early stage and by the use of mammography at least 30\% drop in breast cancer losses [5]. But some of the breast lesions such as micro-calcification, breast masses, shape distortion, and irregularity between breasts may not be detected by screening mammography because it is very difficult to interpret the morphological features [6]. Dense breast parenchyma is highly challenging job for sustaining sensitivity of mammography which depreciates both recognition and classification tasks [7]. In several types of pictures, texture is considered as important attributes. This however, can be a challenging task because the location, size, shape, and texture of a tumor are not constant. Hence it is key necessity to increase the accuracy of screening databases by developing an automatic classification system. This system could be a useful tool for radiologists to screen out doubtful cases, however, the accuracy of lesions classification depends on the extraction of the most significant features.

In the present study, two set of features have been analyzed, Gabor-wavelet features and Statistical features. The first group of the feature extraction is Gabor-wavelet method, which has the ability to yield optimized diverse resolution information in both time and frequency domain [8]. The second group of features is statistical type, which is based on grey scale histogram distribution among pixels. It includes First Order Statistics (FOS) features, Gray Level Cooccurrence Matrix (GLCM), Grey Level Run Length Matrix (GLRLM) and Statistical Features Matrix (SFM) methods. These features are based on the relationship among image pixels. Although, Gabor-wavelet features and statistical features like FOS, SFM and have been widely employed separately or in combination with each other in many different studies, their individual benefits and applicability have not been compared. This motivated the present research to investigate the efficacy and capability of these features in lesions classification.

\section{Related Work:}

Many of previous studies have presented that the performance accuracy for identifying a breast cancer drops due to development of breast thickness [9]. Computer based classification system is needful for detection of breast lesions in dense mammograms. This computer based diagnosis system includes the various texture models. There are many studies which includes the various texture features which are used for classification.

Li et al. [10] have considered the size and location of region of interest (ROI), and also included the effect of size and location. They determined that the location of ROI plays important role for performance of texture features. The accuracy of performance decreases if the location of ROI is 
moved from the midpoint of breast area to the back part of nipple. Because that back part of breast includes the thick or dense region.

Bovis et al. [11] done the classification of mammogram task based on mass and density. The authors used 377 mammograms from DDSM database for their work. They used only four texture models for classification purpose. There are two unlike algorithms which adopt by the authors. First includes the two class problem and second includes the four class problem. ANN classifier used for the classification purpose. They achieved $71.4 \%$ accuracy.

Petroudi et al. [12] includes a method for involuntary classification of breast mass or density arrangements. These breast density arrangements inside the breast are shown by statistical features. In their technique, every mammogram is classified into three unlike parameters: breast tissue, background and pectoral muscle. In conclusion, an overall of 132 mammograms selected from the Oxford Database.

Hapfelmeier et al. [13] evaluated two computer aided diagnosis prototypes which includes segmentation, texture feature extraction, and classification for mass lesions and micro calcification for evaluating the hazard of breast cancer. The result includes classification performance of CAD prototype about $77.7 \%$ for 242 texture features. These analyses included 1347 ROIs on DDSM database. They used Support Vector Machine (SVM) for classification task and Linear Discriminant Analysis (LDA) used for feature selection task.

Oliver et al. [14] suggested a CAD method for classification of breast thickness using morphological and roughness features. They have used two sets of database for evaluating the accuracy of proposed system. First is mini-MIAS database, that includes 322 images and the second is DDSM database, which includes 831 images. They have employed Bayesian classifier, Decision Tree classifier, and K-Nearest Neighbor for classification task and achieved a maximum accuracy of $86 \%$.

There are many of texture features which basically depend upon the neighboring pixels, as Gray Level Co-Occurrence Matrix (GLCM) and Gray Level Run-Length Matrix (GLRLM), Haralick, [15] presented GLCM method in 1973. Many of investigators used the GLCM matrix for extracting the features which are useful for classification. 100 features extracted by Zarchari et al. [16] where they used contour based methods, statistical, GLCM, intensity, and Gabor. Georgiadis et al. [17] extracted 4 different features from histograms, 4 different and 10 features from GLRLM matrix and 22 features from GLCM matrix.

Karssemeijer et al. [18] found 67\% accuracy for BIRADS-IV images using k-NN classifier Sampaio [19] considered 623 images from BIRADS-II image database. Shape and texture features were studied using Cellular neural network and an accuracy of $80 \%$ has been reported.

Sharma et al. [20] concluded the SVM is the best classifier for the classification of breast density and it had an accuracy of $89 \%$ when feature selection is used. But it had been only for 2class problem not more.

Table 1 represents the summary of literature review in chronological order.

Table-1 Summary of previous breast density classification in chronological order

\begin{tabular}{|c|c|c|c|c|c|c|}
\hline Author & $\begin{array}{c}\text { Image } \\
\text { classes }\end{array}$ & $\begin{array}{c}\text { No. of } \\
\text { Images }\end{array}$ & Features & Classifiers & $\begin{array}{c}\text { Accuracy } \\
\text { (\%) }\end{array}$ & Drawback \\
\hline $\begin{array}{c}\text { Karssemeijer } \\
\text { et al. [18] }\end{array}$ & $\begin{array}{c}\text { BIRADS- } \\
\text { IV }\end{array}$ & 615 & $\begin{array}{c}\text { First Order } \\
\text { Statistics }\end{array}$ & k-NN & 67 & $\begin{array}{c}\text { For the classification } \\
\text { number of cases differed } \\
\text { by another classes was } \\
\text { only 2\% }\end{array}$ \\
\hline
\end{tabular}




\begin{tabular}{|c|c|c|c|c|c|c|}
\hline $\begin{array}{c}\text { Bovis, et al. } \\
{[12]}\end{array}$ & $\begin{array}{l}\text { BIRADS- } \\
\text { IV }\end{array}$ & 377 & $\begin{array}{l}\text { SGLD Features, } \\
\text { Fourier transform, } \\
\text { LTEM and DWT } \\
\text { textures }\end{array}$ & ANN & 71.40 & $\begin{array}{c}\text { The four classes } \\
\text { increase the probability } \\
\text { of misperception in the } \\
\text { subsequent } \\
\text { classification. }\end{array}$ \\
\hline $\begin{array}{l}\text { Petroudi, et } \\
\text { al. [13] }\end{array}$ & $\begin{array}{l}\text { BIRADS- } \\
\text { II }\end{array}$ & 132 & $\begin{array}{c}\text { Statistical } \\
\text { Distribution \& } \\
\text { gray level based } \\
\text { features }\end{array}$ & $\begin{array}{l}\text { K-mean } \\
\text { algorithm }\end{array}$ & 76 & $\begin{array}{c}\text { Invariably filter } \\
\text { response is low due to } \\
\text { arithmetical } \\
\text { distribution of } \\
\text { dimensional space. }\end{array}$ \\
\hline Li et. al [10] & $\begin{array}{l}\text { BRCA1/ } \\
\text { BRCA } 2\end{array}$ & 90 & $\begin{array}{c}\text { First order } \\
\text { statistics texture } \\
\text { features, Fourier } \\
\text { and fractal } \\
\text { analysis }\end{array}$ & $\begin{array}{c}\text { Bayesian } \\
\text { artificial } \\
\text { neural network }\end{array}$ & 72 & $\begin{array}{c}\text { Accuracy of } \\
\text { classification } \\
\text { significantly decreased } \\
\text { with decreasing the } \\
\text { ROI size }\end{array}$ \\
\hline $\begin{array}{l}\text { Oliver, et } \\
\text { al.[14] }\end{array}$ & $\begin{array}{l}\text { BIRADS } \\
\text { 4-Class }\end{array}$ & 831 & $\begin{array}{l}\text { Relative areas, } \\
\text { center of mass, } \\
\text { Intensity of both } \\
\text { cluster }\end{array}$ & $\begin{array}{l}\text { kNN, Decision } \\
\text { tree, Bayesian } \\
\text { classifier }\end{array}$ & 77 & $\begin{array}{l}\text { Biasing occurred in } \\
\text { methodology } \\
\text { algorithms }\end{array}$ \\
\hline $\begin{array}{l}\text { Sampaio et } \\
\text { al. [19] }\end{array}$ & $\begin{array}{l}\text { BIRADS- } \\
\text { II }\end{array}$ & 623 & $\begin{array}{c}\text { Shape and texture } \\
\text { features }\end{array}$ & $\begin{array}{c}\text { Cellular neural } \\
\text { network }\end{array}$ & 80 & $\begin{array}{c}\text { Parameters are hard to } \\
\text { understand. Complex } \\
\text { parameters }\end{array}$ \\
\hline $\begin{array}{l}\text { Sharma, et } \\
\text { al. [20] }\end{array}$ & $\begin{array}{c}\text { Fatty and } \\
\text { dense }\end{array}$ & 322 & $\begin{array}{c}\text { SGLCM, GLDS, } \\
\text { FoS, SFM, LTEM }\end{array}$ & SVM & 89 & $\begin{array}{l}\text { Only for 2-class } \\
\text { problem not more }\end{array}$ \\
\hline
\end{tabular}




\section{Database:}

Digital Database for Screening Mammography (DDSM) [21] database has been used for this study, which is publically available at University of South Florida. 480 cases have been used for this study. There are different types of file in DDSM database, first is "ics" file, second is four image files which are using lossless JPEG encoding, third is zero to four overlay files and last one is "16 bit PGM" files. There are two most common methods of breast projection, namely Cranio-Caudal (CC) and Medio Lateral Oblique (MLO). The CC view is taken from above, so area close to the chest wall does not display. In MLO projection, X-ray is taken from front side of the breast as whole breast is visible. In the present work, overall $480(160 \times 3)$ mammograms (MLO views) including of 160 mammograms from every of the 3 categories of breast lesions diagnosis taken from the DDSM database.

\section{Texture based features extraction technique:}

In this study, Gabor wavelet features and Statistical features are considered and compared for classification of mammograms. Statistical features are texture-based feature, which are extracted using techniques like FOS, GLCM, GLRLM and SFM. A brief discussion has been presented in the following section about these features.

\section{(a) Gabor wavelet based feature extraction method}

A common 2-D Gabor filter is originally a sinusoidal function. 2-D Gabor function is modulated by dual dimensional Gaussian function, where ' $\mathrm{W}$ ' is the modulation frequency, can be defined as:

$$
G B(x, y)=g_{\sigma}(x, y) \exp [2 \pi j W(x \cos \theta+y \sin \theta)]
$$

Where

$$
g_{\sigma}(x, y)=\frac{1}{2 \pi \sigma_{x} \sigma_{y}} \exp \left[-\frac{1}{2}\left(\frac{x^{2}}{\sigma_{x}^{2}}+\frac{y^{2}}{\sigma_{y}^{2}}\right)\right]
$$

The parameter $\theta$ signifies the direction parameter, $\sigma$ signifies the scale, and $\mathrm{W}$ is the modulation frequency of the Gaussian function. These types of Gabor functions have many of application like tuning, image compression and classification for texture. When an image $(x$,$) is convoluted$ with a Gabor function $(x$,$) , the result gives a Gabor filtered image.$

The Gabor wavelets are limited with in two-dimensional Gaussian envelope. There are many direction or orientation and scale which are similar to the complex planar. Every Gabor wavelet comprises a specific orientation and wavelength. The Gabor wavelets are usually measured as distinct set of self-similar functions. If $(x$,$) is the mother Gabor wavelet, at that point this self-$ similar filter group is acquired by appropriate rotations and dilations of $(x$,$) . The Discrete Gabor$ Wavelet Transform (DGWT) of image $(x$,$) size (M \times N)$ is termed as:

$$
G_{p q}(x, y)=\sum_{s} \sum_{t} A(x-s, y-t) \cdot \psi_{p q}^{*}(s, t)
$$

Where $s$, are the parameters for size deviation of filter mask; $q$, are the positioning or orientation and the scale values individually. $\psi_{p q}^{*}$ is complex conjugate of $\psi_{p q}$, which is the self-similar function formed from the spin and dilation of the mother wavelet ' $\psi$ ' and is described as: 


$$
\psi(x, y)=\left(\frac{1}{2 \pi \sigma_{x} \sigma_{y}}\right) \exp \left[-\frac{1}{2}\left(\frac{x^{2}}{\sigma_{x}^{2}}+\frac{y^{2}}{\sigma_{y}^{2}}\right)\right] \exp (2 \pi j W x)
$$

Here show the generating functions which constructs the Gabor Wavelet function, defined as:

$$
\psi(x, y)=a^{-p} \psi(\bar{x}, \bar{y})
$$

Where, $q=0,1 \ldots Q-1$, and $p=0,1 \ldots P-1$. Extra factors are the scale factor $a$; the entire number of scales is $P$; overall records of directions are symbolized by $Q$.

$$
\begin{aligned}
& \bar{x}=a^{-p}(x \cos \theta+y \sin \theta) \\
& \bar{y}=a^{-p}(y \cos \theta-x \sin \theta), \text { for } a>1 \text { and } \theta=\frac{q \pi}{Q}
\end{aligned}
$$

In the present study, these Gabor Wavelet Filters (GWT) are acquired at 4 different scales and 6 different angles $\left(30^{\circ}, 60^{\circ}, 90^{\circ}, 120^{\circ}, 150^{\circ}, 180^{\circ}\right)$. 24 GWT is created with these settings of angle and scale. we follow the same convention as shown in Figurel. The convolution between an image and these GWT filters provides 40 Gabor filtered output images. These output Gabor filtered images comprise diverse textural information for classification purpose. The essential parameter energy of each output Gabor filtered image is considered using the subsequent function:

$$
E N_{G W T}(p, q)=\sum_{x} \sum_{y} G_{p q}(x, y)
$$

The mean $\mu_{G W T}$ and standard deviation $\sigma_{G W T}$ for every Gabor filters are defined below. These standards indicate the feature of an identical texture image.

$$
\begin{gathered}
\mu_{G W T}=\frac{E N_{G W T}(p, q)}{M N} \\
\sigma_{G W T}=\sqrt{\frac{\sum_{x} \sum_{y}\left|G_{p q}(x, y)\right|-\mu_{p q}}{M N}}
\end{gathered}
$$
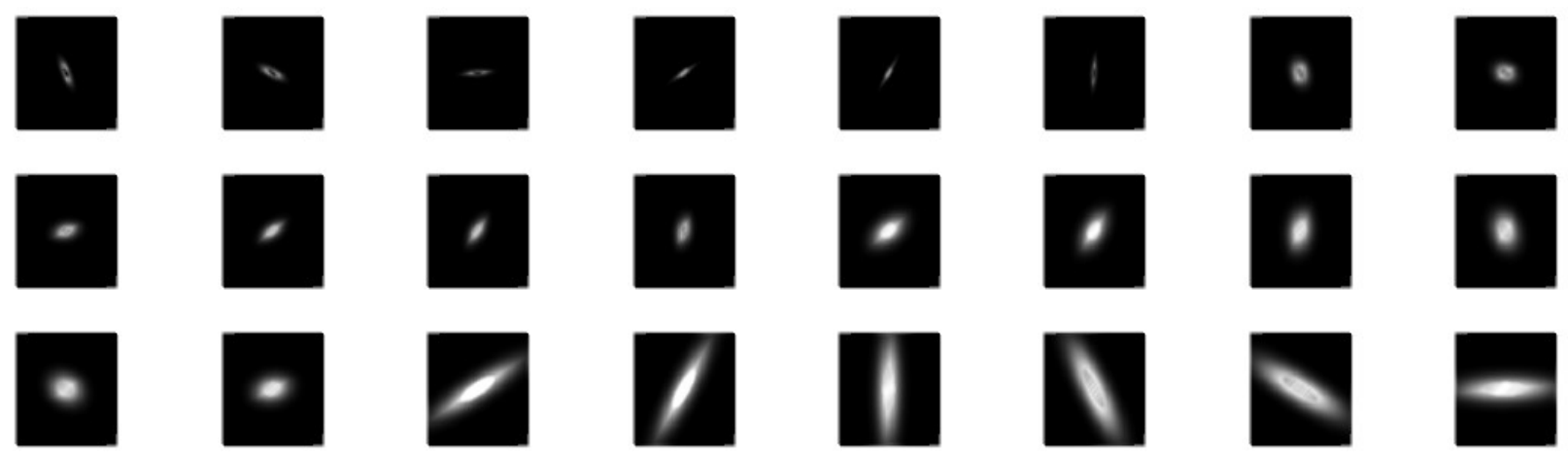

Figure 1 Gabor Wavelet kernel with 4 scales and 6 orientations

\section{(b) Statistical based feature extraction methods:}




\section{(i) First order statistics:}

First order statistical features are determined from the spatial distribution of grey levels values of an image. FOS deals with the individual pixel of an image, not depends upon neighborhood pixels

(ii) Gray Level Co-occurrence matrix (GLCM):

The GLCM is based on the relation between neighboring pixels at different offset and different angles, where first pixel is called as reference and the second is called as neighboring pixel. Gray level co-occurrence matrix is used to compute the texture features based on second order statistics. [15]

\section{(iii) Gray level run length matrix (GLRLM)}

The basic of run-length matrix is proposed by researcher Galloway [22]. Coarse structure or long gray levels run are analyzed by gray level run length matrix. This matrix (GLRLM) is statistical based approach for higher runs of pixels

\section{(iv) Statistical features matrix (SFM):}

The basic of statistical feature matrix is proposed by C.M. Wu [23]. It is used for visual perception based texture feature extraction. Correlation between two statistical feature matrixes is simply defined by measurement of distance. Here we have been extracted four features based on visual perception. Four statistical matrix features are coarseness, contrast, periodicity, and roughness.

\section{Experimental methodology}

\subsection{System Overview}

The proposed automated algorithm includes classification of lesion types, ROI Extraction, feature extraction, dimensionality reduction, classification, and feature efficacy evaluation. A tumor classification approach described below is then applied to every instance of the window. If the window is classified to have tumor, the central pixel of the window will be labeled as tumor. On the other hand, if it is classified as healthy, the central pixel will be labeled as healthy. A proposed post processing method is applied to remove the false positives/negatives. Additionally, we report a study in which we compare the efficiency of Gabor wavelet features with a set of statistical features; i.e., the two main groups of competent and successful texture-based features in tumor classification. Several classification methods such as SVM, KNN, and multilayer perceptron (MLP) are applied for efficacy evaluation of the two feature sets. Final results are then compared using three performance criteria described below. The overall process has been shown in Figure 2 


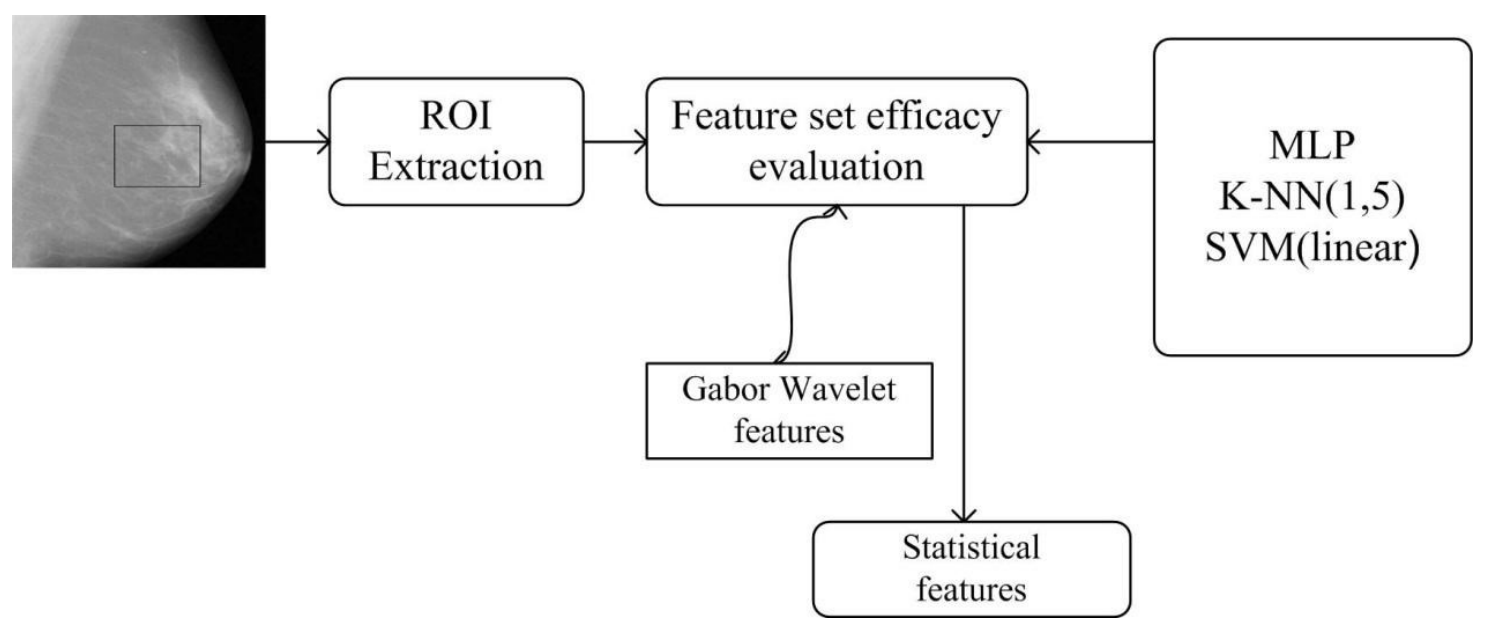

Figure 2 Framework for the proposed system

\subsection{Performance criteria}

The performance of the proposed approach for the classification of normal, benign and cancer mammograms is measured using accuracy. Classification accuracy is depends on the number of samples correctly classified. Higher the accuracy, better the classifier is performing.

$$
\text { Accuracy }=\frac{(T P+T N)}{(T P+F P+T N+F)}
$$

Where, $\mathrm{TP}=$ number of true positives; $\mathrm{FP}=$ number of false positives; $\mathrm{TN}=$ number of true negatives; $\mathrm{FN}=$ number of false negatives

Confusion matrix shows information about actual and predicted classifications successfully completed by the classifier. Here table 2 shows the basic confusion matrix.

Table 2 Confusion matrix

\begin{tabular}{|l|c|c|}
\hline \multirow{2}{*}{ Actual } & \multicolumn{2}{|c|}{ Predicted } \\
\cline { 2 - 3 } & Positive & Negative \\
\hline Positive & TP & FP \\
\hline Negative & FN & TN \\
\hline
\end{tabular}

\subsection{Classification Module}

\subsubsection{Support Vector Machine (SVM)}

SVM can be performed for dual category problems. It categorizes the complex data by defining the finest hyper plane. The hyper plane distributes the plane into two classes the one side of the plane belongs to one class and second side of the plane belongs to other class [24]. Larger width margin boundary gives the best hyperplane between classes 
There are many parameters used for categorization.

- $\in($ For round-off error $)=1.0 \mathrm{E}-12$

- Kernel= Linear kernel

- $\mathrm{C}($ Soft margin constant $)=1$

\subsubsection{Multilayer perceptron (MLP):}

MLP is encouraged by the human brain. There are three basic layers in multilayer perceptron model. MLP is one case of feed-forward neutral network [25]. It associates numerous perceptron (simple neural networks) to generate non-linear decision boundary. There are many of parameters used for classification:

- Momentum $=0.2$

- $\quad$ Learning rate $=0.1$

- Validation threshold $=20$

- Activation function: Sigmoid

- $\quad$ Training time $=500$

\subsection{3. k-NEAREST NEIGHBOURS (k-NN)}

In this approach, classification task is performed based on the type of adjacent neighbor(s), so the method is recognized as nearest neighbor classifier. If there are more than one neighbor this method is known as k-Nearest Neighbors.

There are some parameters which include in k-NN:

- $\mathrm{k}=1,3$ and 5

- Search procedure: Linear nearest adjacent search process.

- Distance function: Euclidean distance

\section{Experimental results and discussion}

\section{Comparing statistical features and Gabor features}

While many techniques for feature extraction have been published, we are not aware of any convincing comparative study in the domain of lesion classification. We have evaluated the proficiency and ability of two widely used feature sets - Gabor wavelets and statistical features in this application. Figure 3 represents the extracted features from statistical and Gabor wavelet. Table 3 to 6 summarize the classification accuracies achieved for different ROI sizes. 


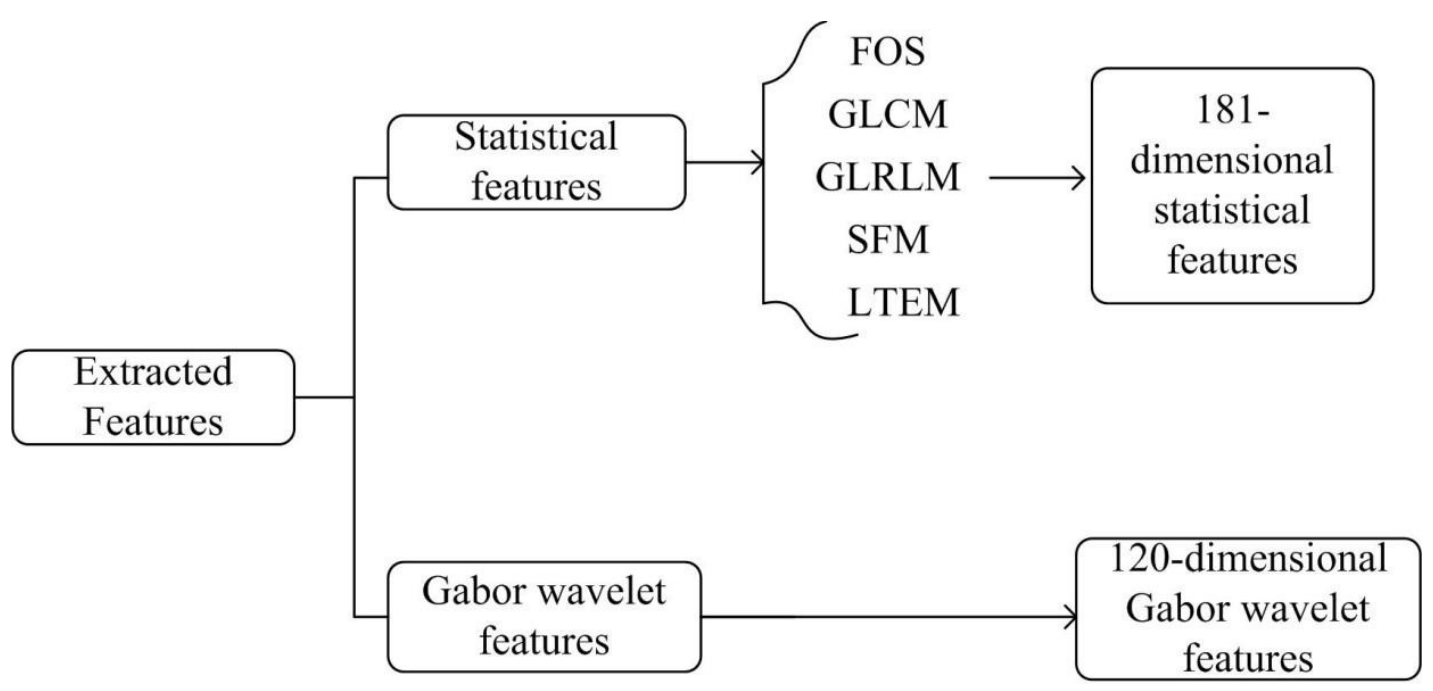

Figure 3 Extracted features

Table 3 Classification accuracies obtained using $64 \times 64$ size ROI dataset. The bold values denote significantly better results than the other set

\begin{tabular}{lllll}
\hline & SVM & MLP $(\mathrm{LR}=0.1)$ & $\mathrm{kNN}(\mathrm{k}=1)$ & $\mathrm{kNN}(\mathrm{k}=5)$ \\
\hline Gabor Wavelet & 48.34 & 43.34 & 54.16 & 60 \\
Statistical features & $\mathbf{5 4 . 1 6}$ & $\mathbf{6 5 . 1 2}$ & $\mathbf{6 5 . 4 7}$ & $\mathbf{6 7 . 3 4}$ \\
\hline
\end{tabular}

Table 4 Classification accuracies obtained using $128 \times 128$ size ROI dataset.

\begin{tabular}{lllll}
\hline & SVM & MLP $(\mathrm{LR}=0.1)$ & $\mathrm{kNN}(\mathrm{k}=1)$ & $\mathrm{kNN}(\mathrm{k}=5)$ \\
\hline Gabor Wavelet & 54.56 & 65.82 & 67.24 & 63.34 \\
Statistical features & $\mathbf{6 4 . 7 2}$ & $\mathbf{7 2 . 3 4}$ & $\mathbf{6 8 . 3 3}$ & $\mathbf{6 5 . 0 0}$ \\
\hline
\end{tabular}

Table 5 Classification accuracies obtained using $256 \times 256$ size ROI dataset.

\begin{tabular}{lllll}
\hline & SVM & MLP $($ LR $=0.1)$ & $\mathrm{kNN}(\mathrm{k}=1)$ & $\mathrm{kNN}(\mathrm{k}=5)$ \\
\hline Gabor Wavelet & 79.50 & 88.00 & 83.60 & $\mathbf{8 0 . 1 3}$ \\
Statistical features & $\mathbf{8 4 . 2 0}$ & $\mathbf{9 3 . 4 0}$ & $\mathbf{8 7 . 2 0}$ & 80.20 \\
\hline
\end{tabular}

Table 6 Classification accuracies achieved on the two different feature sets obtained from $512 \times 512$ size ROI dataset.

\begin{tabular}{lllll}
\hline & SVM & MLP $(\mathrm{LR}=0.1)$ & $\mathrm{kNN}(\mathrm{k}=1)$ & $\mathrm{kNN}(\mathrm{k}=5)$ \\
\hline Gabor Wavelet & 73.50 & 78.00 & 75.00 & $\mathbf{7 0 . 8 3}$ \\
Statistical features & $\mathbf{7 4 . 2 2}$ & $\mathbf{8 3 . 1 4}$ & $\mathbf{7 7 . 2 0}$ & 70.00 \\
\hline
\end{tabular}

It has been observed from the results that statistical features have more accuracy of classification for all three criteria in the case of SVM with linear kernel, Multilayer perceptron and K-NN classifiers. However, Gabor wavelets features lead to better results for $512 \times 512$ and $256 \times 256$ size ROIs by $\mathrm{K}-\mathrm{NN}(\mathrm{K}=5)$ method. Although Gabor wavelets are popularly used in medical imaging due to their directional sensitivity, they consume large computational cost and slow in 
processing. To evaluate this computational cost, the run-times needed for the individual steps have been measured and presented in Table 7.

Table 7 Run time for the individual steps of the algorithm for 40 subjects (for $256 \times 256$ )

\begin{tabular}{lcc}
\hline Algorithm Step & \multicolumn{2}{c}{ Time elapsed (second) } \\
\hline & Gabor features & Statistical features \\
\hline feature extraction time & 300 & $\mathbf{1 8 0}$ \\
SVM Classifier & 0.20 & $\mathbf{0 . 0 9}$ \\
MLP Classifier & 0.45 & $\mathbf{0 . 3 4}$ \\
KNN Classifier & 0.59 & $\mathbf{0 . 0 2}$ \\
\hline
\end{tabular}

\section{Conclusion}

In this paper, a comparative study of Gabor and Statistical features has been done and subsequently, an automated framework is proposed, which can classify mammogram images containing tumor. Three well known classifiers MLP, SVM and KNN have been used in the study. The comparison results show that statistical features have higher accuracy than Gabor wavelet based features. Moreover, statistical features have much smaller dimensionality than Gabor wavelet based feature. Gabor wavelets features occupy a large amount of memory; they are highly redundant and lead to high computational costs. The proposed algorithm is based on statistical features, which is highly accurate (93.4\%) and low in computational complexity. Therefore statistical features are significantly capable to differentiate tumor tissues from other tissue types in mammograms.

\section{Acknowledgement}

The authors are thankful to Digital database for screening mammography research laboratory, for providing the mammogram images. The authors are also thankful to Thapar Institute of Engineering \& Technology, Patiala for providing the necessary infrastructure and support.

\section{References:}

[1] Population Based Cancer Report http://ncrpindia.org/Annual_Reports.aspx. Date: 01/02/2016 [2] Cancer facts and figure. http://www.cancer.org/research/cancerfactsstatistics/Cancerfacts figures2015, Date: 01/02/2016

[3] Kopans D., Breast Imaging (cd 2), Philadelphia: J.B. Lippincott Company, 1989.

[4] Howard J., "Using mammography for cancer control: an unrealized potential," CA: A Cancer Journal for Clinicians, vol. 37, pp. 33-48, 1987.

[5] Cheng H.D., Lui Y.M., and Freimanis R.I., "A novel approach to micro calcification detection using fuzzy logic technique," IEEE Transactions on Medical Imaging, vol.17, pp. 442450, June 1998.

[6] Mousa R., Munib Q., and Moussa A., "Breast Cancer Diagnosis System based on Wavelet Analysis and Fuzzy-Neural," Expert Systems with Applications, vol. 28, pp. 713-723, 2005.

[7] Sampat PM., Markey MK, and Bovik AC., "Computer-aided detection and diagnosis in mammography," Handbook of Image and Video Processing, 2nd ed., A. C. Bovik Ed. Academic Press, pp.1195-1217, 2005. 
[8] Manjunath B.S., and Ma W.Y., "Texture features for browsing and retrieval of image data", IEEE Trans Pattern Anal Mach Intell, vol.18, pp. 837-842, 1996.

[9] Wolfe JN, Breast patterns as an index of risk for developing breast cancer. Am J Roentgenol, vol.6,pp.1130-1137, 1976

[10] Li H., Giger M.L., Huo Z., Olopade O.I., Lan L., Weber B.L., and Bonta I. , "Computerized analysis of mammographic parenchymal patterns for assessing breast cancer risk: effect of ROI size and location", Med Phys vol.31(3), pp.549-555, 2005

[11] Bovis K., and Singh S., "Classification of mammographic breast density using a combined classifier paradigm”, In 4th International Workshop on Digital Mammography, pp.1-4, 2002

[12] Petroudi S, Kadir T, and Brady M "Automatic classification of mammographic parenchymal patterns: a statistical approach", In: proceedings of the 25 th annual international conference of the IEEE on engineering in medicine and biology society

vol.1, pp.798-801, 2003

[13] Hapfelmeier A, and Horsch A "Image feature evaluation in two new mammography CAD prototypes", Int J CARS, vol.6, pp.721-735, 201

[14] Oliver A, Freixenet J, Marti R, Pont J, Pérez E, Denton ER, and Zwiggelaar R, “A novel breast tissue density classification methodology", Information technology in biomedicine. IEEE Trans, vol.12(1): pp.55-65,2008

[15] Haralick R.M., Shanmugam K., and Dinstein I.H., "Textural features for image classification", IEEE Trans Syst Man Cybern, vol.3,pp.610-621, 1973

[16] Zacharaki E I, Wang S, Chawla S, Yoo D S, Wolf R, Mehem E R, and Davatzikos C, "Classification of brain tumor and grade using MRI texture in a Machine Learning technique", Magn. Reson. Med., vol. 62, pp.1609-1618, 2009.

[17] Georgiardis P, Cavouras D, Kalatzis I, Daskalakis A, Kagadis G C, Malamas M, Nikifordis G, and Solomou E, "Improving brain tumor characterization on MRI by probabilistic neural network on non-linear transformation of textural features", Comput Meth Prog bio., vol.89, pp.24-32, 2008.

[18] Karssemeijer N, "Automate classification of parenchymal patterns in mammograms", Physics in medicine and Biology, vol.43, pp.365-389, 1998

[19] Sampaio B., Moraes W., Silva C., and Gattass M., "Detection of masses in mammogram images using CNN, geostatistic functions and SVM," Computers in Biology and Medicine, vol.41, pp. 653-664, 2011

[20] Sharma V., Singh S.,"CFS SMO based classification of using multiple texture models", Medical Biological Engineering and Computing, vol.52, pp. 521-529, 2014

[21] Heath M., Bowyer K., Kopans D., Moore R. and Kegelmeyer P. J.," The digital database for screening mammography." In proceeding. 5th int. workshop on digital mammography. Medical Physics Publishing, pp. 212-218, London, 2001

[22] Galloway M.M., "Texture analysis using gray level run lengths," Comput. Graphics Image Process. , vol.4, 172-179, 1975.

[23] Wu C.M., and Chen Y.C., "Statistical feature matrix for texture analysis" Comput Vis Graph Image Process, vol. 54(5), pp. 407-419, 1992

[24] Support Vector Machines (SVM), http://in.mathworks.com/help/stats/supportvectormachines-svm.html?refresh=true, Date: 15 Dec 2015.

[25] Tadiou K.M., "Artificial Neural Networks "http://futurehumanevolution.com/artificial"intelligence-future-humanevolution/ artificial-neural-networks, 2014. 